\title{
Quality-of-Life Comparison of Dapagliflozin Versus Dipeptidyl Peptidase 4 Inhibitors in Patients with Type 2 Diabetes Mellitus: A Randomized Controlled Trial (J-BOND Study)
}

\author{
Hitoshi Ishii (D) - Hiroki Nakajima · Nozomu Kamei · Tetsuji Niiya • \\ Toru Hiyoshi · Yuko Hiramori · Shigeyuki Ohtsu • Takashi Noto • \\ Dai Shimono
}

Received: September 7, 2020 / Accepted: October 3, 2020 / Published online: October 15, 2020

(C) The Author(s) 2020

\section{ABSTRACT}

Introduction: No study has compared the effects of sodium-glucose cotransporter 2 inhibitors (SGLT2is) and dipeptidyl peptidase 4 inhibitors (DPP4is) on patients' quality-of-life (QOL).

Methods: We enrolled 253 drug-naïve Japanese patients with type 2 diabetes mellitus (T2DM), randomly assigned them into a dapagliflozin (SGLT2i) group or DPP4i group in

Electronic Supplementary Material The online version of this article (https://doi.org/10.1007/s13300020-00941-8) contains supplementary material, which is available to authorized users.

H. Ishii $(\square)$

Department of Doctor-Patient Relationships, Nara Medical University, Kashihara, Nara, Japan

e-mail: hit3910@gmail.com

H. Nakajima

Department of Diabetes and Endocrinology, Nara Medical University, Kashihara, Nara, Japan

N. Kamei

Hiroshima Red Cross Hospital and Atomic-Bomb Survivors Hospital, Hiroshima, Hiroshima, Japan

T. Niiya

Matsuyama Shimin Hospital, Matsuyama, Ehime, Japan

T. Hiyoshi

Division of Diabetes and Endocrinology, Japanese

Red Cross Medical Center, Shibuya-ku, Tokyo, Japan approximately $1: 1$ ratio, and monitored them for 24 weeks. The primary endpoint was the proportion of subjects indicating improvement in the "overall quality of life" domain of SHIELD-WQ-9 at week 24. Secondary endpoints included other domains of SHIELD-WQ-9, DTRQOL, EQ-5D-5L, medication preference, medication adherence, diet therapy adherence, body weight, body mass index (BMI), abdominal circumference, HbA1c, and frequency of adverse events.

Results: The proportion of subjects indicating improvement in the "overall quality of life" domain of SHIELD-WQ-9 at week 24 was higher in the dapagliflozin group $(28.4 \%)$ than in the DPP4i group $(18.6 \%)(p=0.08)$. The proportion

\author{
Y. Hiramori \\ Suzuran-Naika Clinic of Medicine, Nara, Nara, Japan \\ S. Ohtsu \\ Nakanoshima Diabetes Clinic, Kawasaki, Kanagawa, \\ Japan \\ T. Noto \\ Nishiyamato Diabetes Clinic, Kita-Katsuragi-gun, \\ Nara, Japan \\ D. Shimono \\ Futata Tetsuhiro Clinic, Fukuoka, Fukuoka, Japan
}


of subjects indicating improvement in the "physical health" domain of SHIELD-WQ-9 at week 24 was significantly higher in the dapagliflozin group (42.2\%) than in the DPP4i group (23.7\%) $(p=0.004)$. Total scores and domain 1 scores of DTR-QOL showed greater improvement in the dapagliflozin group $(14.3 \pm 15.6$ and $15.5 \pm 20.8$, respectively) than in the DPP4i group (10.2 \pm 15.6 and $10.3 \pm 19.5$, respectively) (both $p=0.05$ ). EQ-5D-5L scores had significantly improved in the DPP4i group $(0.023 \pm 0.088)(p=0.005)$; the intergroup difference was not significant $(p=0.14)$. Body weight $(p<0.001)$, BMI $(p<0.001), \quad$ and abdominal circumference $(p=0.019)$ had significantly decreased in the dapagliflozin group compared with the corresponding values in the DPP4i group.

Conclusion: Dapagliflozin showed a comparable or more favorable benefit on Japanese patients' QOL compared with DPP4is. Dapagliflozin was well tolerated. It significantly reduced body weight, which was significantly correlated with improvement in the patients' QOL. This study demonstrates that dapagliflozin can be used as a first-line drug for T2DM in Japan with a beneficial impact on patients' QOL.

Trial Registration: University Hospital Medical Information Network Clinical Trial Registry (UMIN000030514); Japan Registry of Clinical Trials (jRCTs051180165).

Keywords: Dapagliflozin;

Dipeptidyl peptidase 4 inhibitor; Quality of life; Sodium-glucose cotransporter 2 inhibitor; Type 2 diabetes mellitus

\section{Key Summary Points}

\section{Why carry out this study?}

No study has compared the effects of sodium-glucose cotransporter 2 inhibitors (SGLT2is) and dipeptidyl peptidase 4 inhibitors (DPP4is) on patients' quality of life (QOL).

This study enrolled drug-naïve patients with type 2 diabetes mellitus and compared the effects of dapagliflozin, an SGLT2i, and DPP4is on patients' QOL.

\section{What was learned from the study?}

Dapagliflozin showed a comparable or more favorable impact on patients' QOL than DPP4is.

The reduction in body weight was significantly correlated with improvement in patients' QOL by dapagliflozin.

The results of this study demonstrate that dapagliflozin can be used as a first-line drug for T2DM, with a beneficial impact on patients' QOL.

\section{DIGITAL FEATURES}

This article is published with digital features, including a summary slide, to facilitate understanding of the article. To view digital features for this article go to https://doi.org/10.6084/ m9.figshare.13042037.

\section{INTRODUCTION}

The American Diabetes Association (ADA) and the European Association for the Study of Diabetes (EASD) recommend metformin as first-line therapy for type 2 diabetes mellitus (T2DM) with comprehensive lifestyle management $[1,2]$. Other oral hypoglycemic agents (OHAs), such as sodium-glucose cotransporter 2 
inhibitors (SGLT2is) and glucagon-like peptide 1 (GLP-1) receptor agonists, are to be used as second-line or further lines of therapy on the basis of cardiovascular or renal risks and glycemic control $[1,2]$. In contrast, the Japanese Clinical Practice Guideline for Diabetes does not define any first-line drug; it recommends the selection of antidiabetic agents on the basis of the individual's pathological condition and risk of hypoglycemia [3, 4]. Because of their effectiveness and low risk of hypoglycemia [5], especially in East Asian populations [6], dipeptidyl peptidase 4 inhibitors (DPP4is) have become the most commonly used OHAs in Japan [7]. However, studies have reported benefits of SGLT2is such as weight reduction $[8,9]$ as well as cardiovascular and renal protection [10-13], with low risk of hypoglycemia $[14,15]$. A clinical trial comparing SGLT2is and DPP4is reported greater reduction in fasting plasma glucose and body weight in the SGLT2i group than in the DPP4i group, although the frequency of hypoglycemic events was similar in both groups [16].

A "patient-centered approach" [1, 2, 17], as recommended by the ADA/EASD, is important for developing a treatment strategy and should consider medication preference and quality-oflife (QOL) of patients. Although both DPP4i $[18,19]$ and SGLT2i [20-23] improve treatment satisfaction and QOL, no study has compared the effects of DPP4is and SGLT2is on patients' QOL. Thus, this Japanese T2D study for evaluating benefits of dapagliflozin, as new first-line therapy, to improve QOL (J-BOND study) enrolled drug-naïve patients with T2DM who had been newly initiated on OHA therapy and compared the effects of dapagliflozin, an SGLT2i, and DPP4is on their QOL. Since many kinds of DPP4is are used in real-world situations, all once-daily DPP4is were used as the control agents in this study, and the effects of dapagliflozin and all kinds of once-daily DPP4is on the QOL were compared.

\section{METHODS}

\section{Study Design}

The J-BOND study was a prospective, randomized, open-label, blinded-endpoint trial, conducted across 42 medical institutions (Supplementary Table 1) in Japan between February 2018 and January 2020. This study and its protocols were first approved by the institutional review board of each participating institution (Supplementary Material) according to the Ethical Guidelines for Medical and Health Research Involving Human Subjects issued by the Ministry of Health, Labour and Welfare in Japan. Following enforcement of the Clinical Trials Act in April 2018, this study and its protocols were again inspected and approved by the Nara Medical University Certified Review Board, which had obtained certification from the Minister of Health, Labour and Welfare in Japan. This study was first registered in the University Hospital Medical Information Network Clinical Trial Registry (UMIN-CTR) (registration number UMIN000030514) and then in the Japan Registry of Clinical Trials (jRCT) (registration number jRCTs051180165) after approval from the certified review board according to the Clinical Trials Act. The study was conducted in accordance with the Declaration of Helsinki, Ethical Guidelines for Medical and Health Research Involving Human Subject, the Clinical Trials Act, and other current legal regulations in Japan. Written informed consent was obtained prior to treatment from all enrolled patients who met the eligibility criteria. All authors had access to the study data and reviewed and approved the final manuscript.

\section{Patient Population}

Patients with T2DM who were initiating therapy with OHAs were included in this study. The inclusion criteria were as follows: (1) patients with T2DM who had newly started therapy with OHAs, (2) HbA1c $\geq 6.5 \%$ at the time of consent, (3) body mass index (BMI) $\geq 23 \mathrm{~kg} / \mathrm{m}^{2}$, (4) 20 years $\leq$ age $<75$ years, and (5) written 
consent. The exclusion criteria were as follows: (1) history of any antidiabetic medication within the last 3 months, (2) history of severe hypoglycemia within a year, (3) type 1 diabetes mellitus or secondary diabetes, (4) perioperative period or with severe infection or severe physical injury, (5) moderate to severe heart failure (New York Heart Association at class III or higher), (6) moderate-to-severe renal disease (estimated glomerular filtration rate $<45 \mathrm{~mL}$ / $\min / 1.73 \mathrm{~m}^{2}$ ), (7) severe liver disease (aspartate aminotransferase $\geq 100 \mathrm{IU} / \mathrm{L}$ ), (8) addiction to alcohol or a drug, (9) possibly pregnant, pregnant, or planning to be pregnant during the study period, (10) dementia, (11) contraindication of the study drug, and (12) other conditions that the investigator/researcher considered inappropriate for the study.

\section{Randomization and Study Intervention}

After obtaining informed consent, eligible patients were randomly assigned to either the dapagliflozin group or the DPP4i group at a ratio of approximately $1: 1$. Randomization was performed using a computer-based dynamic allocation method with a minimization procedure to balance two allocation factors (HbA1c and BMI) across the groups. Subjects assigned to the dapagliflozin and DPP4i groups started to use $5 \mathrm{mg}$ of dapagliflozin per day or DPP4i (sitagliptin, vildagliptin, alogliptin, linagliptin, teneligliptin, anagliptin, or saxagliptin), respectively, at an initial dose defined in the package insert of each product. Since many kinds of DPP4is are used in real-world situation, all once-daily DPP4is were used as the control agents in this study. To compare medication adherence and preference, once-weekly DPP4is and twice-daily DPP4is were not included in this study. Subjects were followed up for 24 weeks, with observation time points at baseline, week 12, and week 24 .

\section{Study Outcomes}

The primary endpoint was the proportion of subjects indicating improvement in the "overall quality of life" domain of SHIELD-WQ-9 at week 24. SHIELD-WQ-9 (Study to Help Improve Early evaluation and management of risk factors Leading to Diabetes Weight Questionnaire-9) is a specific questionnaire to assess health-related QOL associated with weight change to determine how weight change has affected nine aspects of daily life: physical health, interaction with family, work performance, interaction with coworkers/friends, social activities, self-esteem, daily activities, emotional health, and overall QOL [24]. Responses to each domain include four options: worsened, improved, stayed the same, and not applicable. The secondary endpoints included the proportion of patients indicating improvement in other domains (physical health, interactions with family, work performance, interactions with coworkers/friends, social activities, daily activities, self-esteem, and emotional health) of SHIELD-WQ-9 at week 24, change in Diabetes Therapy-Related QOL (DTR-QOL) and EuroQol five-dimensional five level version (EQ-5D-5L) scores, medication preference, medication adherence, diet therapy adherence, body weight, BMI, abdominal circumference, HbA1c, and frequency of adverse events. DTR-QOL is a diabetes-specific questionnaire with four domains: domain 1 , burden on social activities and daily activities; domain 2 , anxiety and dissatisfaction with treatment; domain 3, hypoglycemia; and domain 4, treatment satisfaction [25]. The each item in the DTR-QOL is a 7-point Likert scale (1, strongly agree; 7 , strongly disagree). The score of each item was reversed so that 7 represented the highest QOL. The total score and each domain score was calculated by addition scores of the attribute items and the conversion of scoring range to $0-100$, so that 0 and 100 represents the lowest and highest QOL, respectively. EQ-5D-5L is a generic questionnaire to assess health-related QOL in five dimensions-mobility, self-care, usual activities, pain/discomfort, and anxiety/depressionwith five levels of severity: no problems, slight problems, moderate problems, severe problems, and extreme problems [26]. The answer to each dimension is converted to a 5-point Likert scale (1, no problems; 5 , extreme problems), and EQ5D-5L score is calculated using standard value sets for Japanese EQ-5D-5L based on the 
composite time trade-off valuation method [27]. Higher EQ-5D-5L score indicates better QOL. Medication preference was investigated by a questionnaire asking "prefer SGLT2i", "prefer DPP4i", or "neither" at each visit after full explanation of advantages and disadvantages of SGLT2i and DPP4i from the investigators. Medication adherence for the study agent throughout the study was calculated as the frequency of actual administration relative to the total planned administration. Diet therapy adherence was investigated by a questionnaire asking "1. not conducted at all", "2. rarely conducted", "3. conducted about half of planned", "4. almost conducted", or "5. perfectly conducted". The numbering of each option was used as the score for diet therapy adherence. The patient-reported outcomes (PROs) (SHIELDWQ-9, DTR-QOL, EQ-5D-5L, medication preference, medication adherence, and diet therapy adherence) were answered by the study subjects by themselves on the paper questionnaire. Other clinical outcomes were collected by the paper case report forms from the investigators.

\section{Sample Size Calculation and Statistical Analysis}

On the basis of the results of a previous study [20], we assumed the proportion of subjects indicating improvement in the "overall quality of life" domain of SHIELD-WQ-9 to be $29.3 \%$ in the dapagliflozin group in this study, which was comparable with that in the dapagliflozin group in the previous study, and $14.0 \%$ in the DPP4i groups in this study, which was comparable with that in the placebo group in the previous study. Under this assumption, 113 subjects per group would provide a power of over $80 \%$ to detect an intergroup difference using a twosided $t$ test at $5 \%$ significance. The dropout rate was estimated to be approximately $10 \%$. Thus, 126 subjects were required per group, yielding a total sample size of 252 .

Analyses for the primary and secondary endpoints were performed on the full analysis set (FAS), which included all subjects assigned to a study intervention. However, subjects with a significant study protocol violation (e.g., registration without consent or registration out of the enrollment period) were excluded from the FAS. Sensitivity analysis was performed with the per-protocol set by excluding subjects with a protocol violation such as violation of eligibility criteria, use of prohibited drugs, or poor medication adherence to the study agent or control agent $(<75 \%$ or $>120 \%)$. Safety analysis included all treated subjects. All tests were twosided, and a $p<0.05$ was considered statistically significant. The number and proportion of subjects who met the criteria were calculated for analysis of the primary endpoint, and the chisquare test was performed for intergroup comparison. Summary statistics for measurement and change from baseline were calculated for the analysis of secondary endpoints, and onesample $t$ test for intragroup comparison and two-sample $t$ test for intergroup comparison were performed for continuous variables. The chi-square test or Wilcoxon signed-rank test was performed for categorical variables. Medication adherence for the study agent throughout the study was calculated as the frequency of actual administration relative to the total planned administration. Summary statistics were calculated, and Fisher's exact test was used for intergroup comparisons of frequency of adverse events. Correlation analysis was performed with Pearson's correlation coefficient and Spearman's rank correlation coefficient. The SAS statistical software package (version 9.4, SAS, Cary, NC, USA) was used to perform all statistical analyses. To avoid bias and ensure quality, data collection, data management, and statistical analyses were performed by third-party entities (Soiken Inc., Osaka, Japan).

\section{RESULTS}

\section{Baseline Characteristics of Study Participants}

A total of 4247 patients were screened, and 3994 patients were excluded from the study. Of these 3994 patients, 3842 did not meet the eligibility criteria, 100 did not provide consent, and 52 were excluded for other reasons. Thus, 253 subjects were registered and randomly allocated 


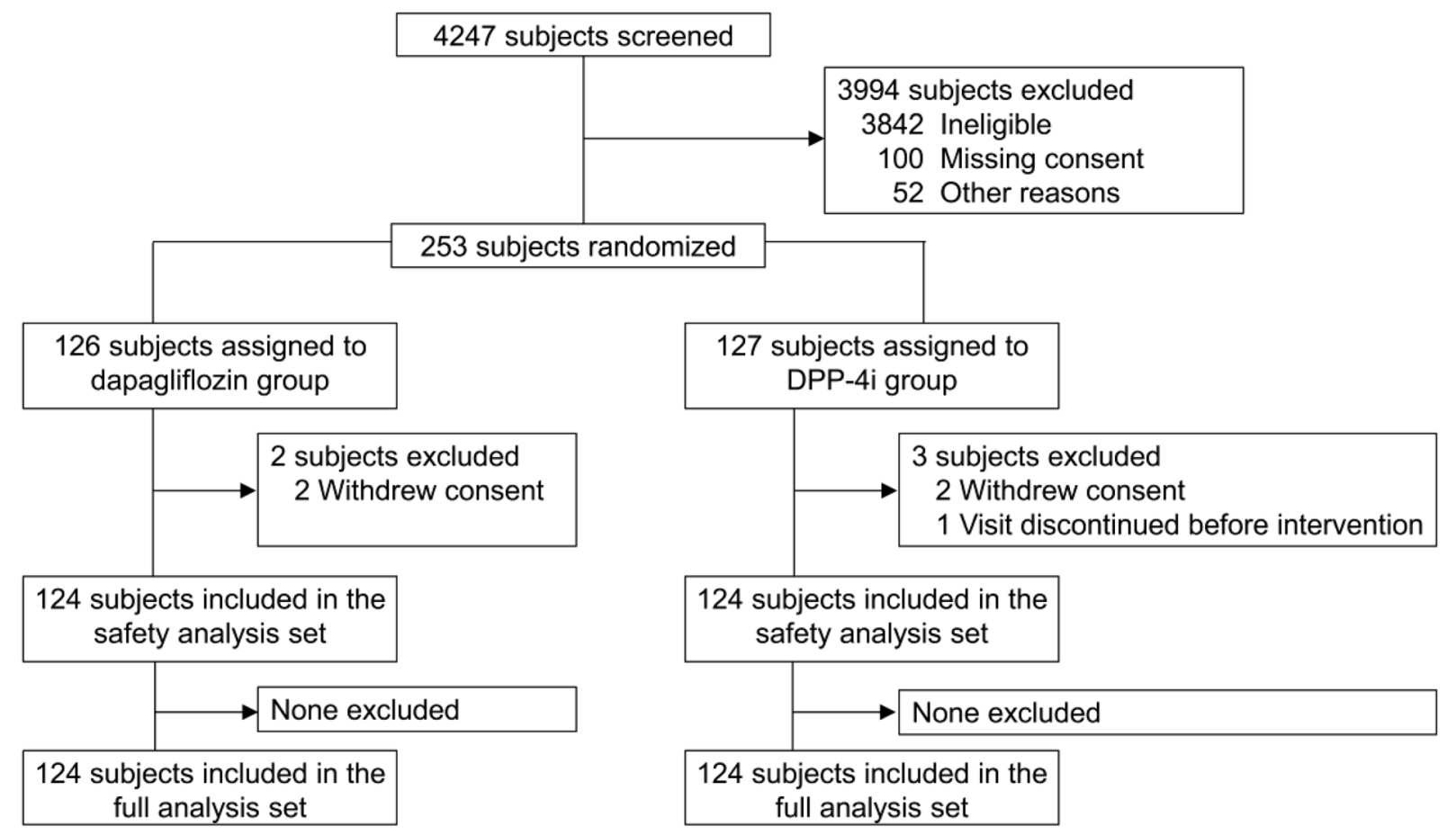

Fig. 1 Study flow chart showing subject enrollment, allocation, and analysis. DPP4i dipeptidyl peptidase inhibitor

to the treatment groups. Although the target sample size was set to 252 , as described in the "Methods", because the last two eligible candidates were registered at the same time, both were included in this study. This issue was reported to and approved by the certified review board. Because of consent withdrawal or visit discontinuation before the start of the study intervention, two and three patients were excluded from the dapagliflozin and DPP4i groups, respectively. As a result, 124 subjects were included in the safety analysis set, and both groups were considered as the FAS (Fig. 1). Of these, because one withdrew consent, and eight in the dapagliflozin group and four in the DPP4i group discontinued the study, 235 subjects (115 subjects in the dapagliflozin group and 120 subjects in the DPP4i group) completed the study.

The baseline characteristics of the subjects are summarized in Table 1 . No significant intergroup differences in baseline characteristics were found, except in the proportion of smokers.

\section{Patient-Reported Outcomes}

\section{SHIELD-WQ-9}

SHIELD-WQ-9 is a specific questionnaire to assess health-related QOL associated with weight change to determine how weight change has affected nine aspects of daily life: physical health, interaction with family, work performance, interaction with coworkers/friends, social activities, daily activities, self-esteem, emotional health, and overall QOL [24]. The number of subjects indicating improvement in the "overall quality of life" domain of SHIELDWQ-9 at week 24 was 31 (28.4\%) and 22 (18.6\%) in the dapagliflozin and DPP4i groups, respectively. Although the proportion was higher in the dapagliflozin group than in the DPP4i group, no significant intergroup difference was observed ( $p=0.08)$ (Table 2$)$. Additionally, the proportion of subjects indicating improvement in the "physical health" domain at week 24 was significantly higher in the dapagliflozin group $(46,42.2 \%)$ than in the DPP4i group $(28,23.7 \%)$ $(p=0.004)$. Other domains did not show any significant intergroup difference. 
Table 1 Baseline characteristics of subjects

\begin{tabular}{|c|c|c|c|}
\hline Characteristic & Dapagliflozin group & DPP4i group & $p$ value \\
\hline Age (years) & $54.9 \pm 11.9(n=124)$ & $53.6 \pm 11.7(n=124)$ & 0.38 \\
\hline Sex (male/female) & $71(57.3 \%) / 53(42.7 \%)$ & $77(62.1 \%) / 47(37.9 \%)$ & 0.52 \\
\hline HbAlc (\%) & $7.8 \pm 1.4(n=119)$ & $8.0 \pm 1.6(n=120)$ & 0.48 \\
\hline Body weight $(\mathrm{kg})$ & $76.6 \pm 16.1(n=123)$ & $76.4 \pm 15.3(n=124)$ & 0.96 \\
\hline Body mass index $\left(\mathrm{kg} / \mathrm{m}^{2}\right)$ & $28.4 \pm 5.0(n=123)$ & $28.3 \pm 4.5(n=124)$ & 0.84 \\
\hline Abdominal circumference $(\mathrm{cm})$ & $97.2 \pm 12.3(n=113)$ & $97.1 \pm 11.5(n=119)$ & 0.95 \\
\hline Duration of diabetes (years) & $3.5 \pm 5.4$ & $3.1 \pm 4.1$ & 0.60 \\
\hline \multicolumn{4}{|l|}{ Smoking habit } \\
\hline No & $75(60.5)$ & $54(43.5)$ & 0.013 \\
\hline Currently & $31(25.0)$ & $36(29.0)$ & \\
\hline Previously & $18(14.5)$ & $34(27.4)$ & \\
\hline Drinking habit & $59(47.6)$ & $69(55.6)$ & 0.25 \\
\hline Renal complications & $1(0.8)$ & $7(5.6)$ & 0.07 \\
\hline Hepatic complications & $21(16.9)$ & $24(19.4)$ & 0.74 \\
\hline Cerebrovascular complications & $1(0.8)$ & $1(0.8)$ & 1.00 \\
\hline Cardiovascular complications & $5(4.0)$ & $2(1.6)$ & 0.45 \\
\hline Hypertension & $56(45.2)$ & $48(38.7)$ & 0.37 \\
\hline Dyslipidemia & $68(54.8)$ & $66(53.2)$ & 0.90 \\
\hline Other complications & $45(36.3)$ & $55(44.4)$ & 0.24 \\
\hline Antidiabetic drugs & $0(0.0)$ & $0(0.0)$ & - \\
\hline Antihypertensive drugs & $41(33.1)$ & $34(27.4)$ & 0.41 \\
\hline Lipid-lowering drugs & $40(32.3)$ & $40(32.3)$ & 1.00 \\
\hline Antithrombotic drugs & $8(6.5)$ & $2(1.6)$ & 0.10 \\
\hline
\end{tabular}

Data are presented as mean \pm standard deviation $(n)$ or frequency (percentage), as appropriate. Student's $t$ test and Fisher's exact test were used to calculate $p$ values for intergroup comparisons for continuous and categorical variables, respectively $D P P 4 i$ dipeptidyl peptidase inhibitor

\section{DTR-QOL}

DTR-QOL is a diabetes-specific questionnaire with four domains: domain 1 , burden on social activities and daily activities; domain 2 , anxiety and dissatisfaction with treatment; domain 3, hypoglycemia; and domain 4, treatment satisfaction [25]. DTR-QOL total scores significantly increased from baseline to week 24 in both groups: changes from baseline were $14.3 \pm 15.6$ $(p<0.001)$ and $10.2 \pm 15.6(p<0.001)$ in the dapagliflozin and DPP4i groups, respectively (Table 3). The degree of increase tended to be higher in the dapagliflozin group than in the DPP4i group $(p=0.05)$, although this was not statistically significant. All scores of domains 1 to 4 significantly increased from baseline to 
Table 2 Proportion of subjects indicating improvement in each domain of SHIELD-WQ-9 at week 24

\begin{tabular}{|c|c|c|c|c|c|}
\hline \multirow[t]{2}{*}{ Proportion of subjects indicating improvement } & \multicolumn{2}{|c|}{ Dapagliflozin group } & \multicolumn{2}{|c|}{ DPP4i group } & \multirow{2}{*}{$\begin{array}{l}\text { Chi-square test } \\
p \text { value }\end{array}$} \\
\hline & $\bar{n}$ & $n(\%)$ & $\bar{n}$ & $n(\%)$ & \\
\hline Physical health & 109 & $46(42.2)$ & 118 & $28(23.7)$ & 0.003 \\
\hline Interactions with family & 109 & $9(8.3)$ & 118 & $8(6.8)$ & 0.67 \\
\hline Work performance & 109 & $12(11.0)$ & 118 & $11(9.3)$ & 0.67 \\
\hline Interactions with coworkers/friends & 109 & $5(4.6)$ & 118 & $5(4.2)$ & $0.90^{*}$ \\
\hline Social activities & 109 & $7(6.4)$ & 118 & $7(5.9)$ & 0.88 \\
\hline Daily activities & 109 & $21(19.3)$ & 118 & $19(16.1)$ & 0.53 \\
\hline Self-esteem & 109 & $13(11.9)$ & 118 & $10(8.5)$ & 0.39 \\
\hline Emotional health & 109 & $23(21.1)$ & 118 & $17(14.4)$ & 0.19 \\
\hline Overall quality of life & 109 & $31(28.4)$ & 118 & $22(18.6)$ & 0.08 \\
\hline
\end{tabular}

Data are presented as frequency (percentage); $p$ values for intergroup comparisons were obtained by using chi-square test. ${ }^{*}$ Does not meet the requirement of chi-square test and $p$ value therefore lacks reliability

DPP4i dipeptidyl peptidase inhibitor, SHIELD-WQ-9 Study to Help Improve Early evaluation and management of risk factors Leading to Diabetes Weight Questionnaire-9

week 24 in both groups (all $p$ values for intragroup comparisons $<0.001$ in all domains in both groups). The degree of increase in domain 1 scores tended to be higher in the dapagliflozin group than in the DPP4i group $(p=0.05)$; however, this did not reach statistical significance. No other significant differences or tendency of difference were observed in intergroup comparisons of the domain scores.

\section{EQ-5D-5L}

EQ-5D-5L is a generic questionnaire to assess health-related QOL in five dimensions-mobility, self-care, usual activities, pain/discomfort, and anxiety/depression [26]. EQ-5D-5L scores increased significantly from baseline to week 24 in the DPP4i group, but not in the dapagliflozin group: changes from baseline were $0.023 \pm 0.088 \quad(p=0.005)$ and $0.005 \pm 0.096$ $(p=0.57)$ in the DPP4i and dapagliflozin groups, respectively (Table 4). However, no significant intergroup difference was observed $(p=0.14)$. EQ-5D-5L visual analogue scale (VAS) increased significantly from baseline to week 24 in both groups: changes from baseline were $4.9 \pm 16.1 \quad(p=0.002) \quad$ and $\quad 5.4 \pm 14.1$ $(p<0.001)$ in the dapagliflozin and DPP4i groups, respectively. No significant intergroup difference was observed $(p=0.81)$.

\section{Medication Preference, Medication Adherence, and Diet Therapy Adherence}

The medication preference differed between the groups at every visit $(p=0.007$ and $<0.001$ at baseline and week 24, respectively) (Table 5). The proportion of subjects indicating "prefer assigned study agent" increased in both groups. Medication adherence throughout the study was comparable between the groups: $91.9 \pm 14.8 \%$ and $94.1 \pm 9.6 \%$ in the dapagliflozin and DPP4i groups, respectively $(p=0.16)$. Both at baseline and week 24 , the median of diet therapy adherence was 3 , and no significant intergroup difference was observed $(p=0.74$ and 0.56 at baseline and week 24 , respectively).

\section{Clinical Outcomes}

Body weight significantly decreased in the dapagliflozin group, but not in the DPP4i group from baseline to week 24: change from baseline 
Table 3 DTR-QOL scores

\begin{tabular}{|c|c|c|c|c|c|c|}
\hline & \multirow[t]{2}{*}{ Week } & \multicolumn{2}{|c|}{ Dapagliflozin group } & \multicolumn{2}{|c|}{ DPP4i group } & \multirow{2}{*}{$\begin{array}{l}t \text { test } \\
p \text { value }\end{array}$} \\
\hline & & $n$ & Mean $\pm S D$ & $n$ & Mean \pm SD & \\
\hline \multicolumn{7}{|l|}{ Total score } \\
\hline \multirow[t]{2}{*}{ Score } & 0 & 123 & $62.9 \pm 16.9$ & 121 & $65.4 \pm 14.8$ & 0.21 \\
\hline & 24 & 108 & $77.2 \pm 15.3$ & 119 & $75.9 \pm 14.9$ & 0.52 \\
\hline Change from baseline & 24 & 108 & $\begin{array}{l}14.3 \pm 15.6 \\
(p<0.001)\end{array}$ & 116 & $\begin{array}{l}10.2 \pm 15.6 \\
(p<0.001)\end{array}$ & 0.05 \\
\hline \multicolumn{7}{|l|}{ Domain 1} \\
\hline \multirow[t]{2}{*}{ Score } & 0 & 123 & $66.0 \pm 21.5$ & 123 & $70.6 \pm 18.9$ & 0.07 \\
\hline & 24 & 108 & $81.4 \pm 16.6$ & 120 & $81.1 \pm 16.0$ & 0.88 \\
\hline Change from baseline & 24 & 108 & $\begin{array}{l}15.5 \pm 20.8 \\
(p<0.001)\end{array}$ & 119 & $\begin{array}{l}10.3 \pm 19.5 \\
(p<0.001)\end{array}$ & 0.05 \\
\hline \multicolumn{7}{|l|}{ Domain 2} \\
\hline \multirow[t]{2}{*}{ Score } & 0 & 123 & $57.9 \pm 21.8$ & 121 & $59.4 \pm 18.8$ & 0.57 \\
\hline & 24 & 109 & $72.6 \pm 21.5$ & 120 & $71.6 \pm 20.7$ & 0.72 \\
\hline Change from baseline & 24 & 109 & $\begin{array}{l}14.1 \pm 19.9 \\
(p<0.001)\end{array}$ & 117 & $\begin{array}{l}12.1 \pm 20.6 \\
(p<0.001)\end{array}$ & 0.45 \\
\hline \multicolumn{7}{|l|}{ Domain 3} \\
\hline \multirow[t]{2}{*}{ Score } & 0 & 123 & $74.5 \pm 24.0$ & 122 & $74.2 \pm 23.6$ & 0.94 \\
\hline & 24 & 110 & $87.0 \pm 19.4$ & 120 & $82.8 \pm 19.3$ & 0.10 \\
\hline Change from baseline & 24 & 110 & $\begin{array}{l}12.9 \pm 23.8 \\
(p<0.001)\end{array}$ & 118 & $\begin{array}{l}9.0 \pm 25.3 \\
(p<0.001)\end{array}$ & 0.23 \\
\hline \multicolumn{7}{|l|}{ Domain 4} \\
\hline \multirow[t]{2}{*}{ Score } & 0 & 123 & $51.2 \pm 17.9$ & 121 & $52.3 \pm 16.4$ & 0.62 \\
\hline & 24 & 110 & $62.0 \pm 23.4$ & 119 & $61.7 \pm 22.8$ & 0.92 \\
\hline Change from baseline & 24 & 110 & $\begin{array}{l}10.2 \pm 26.5 \\
(p<0.001)\end{array}$ & 116 & $\begin{array}{l}9.4 \pm 23.1 \\
(p<0.001)\end{array}$ & 0.81 \\
\hline
\end{tabular}

Data are presented as mean \pm standard deviation (SD); $p$ values for intragroup comparisons were obtained by using onesample $t$ test. Intergroup comparisons were performed by using two-sample $t$ test DPP4i dipeptidyl peptidase inhibitor, DTR-QOL Diabetes Therapy-Related Quality of Life

was $-2.6 \pm 2.5 \mathrm{~kg}(p<0.001)$ and $0.3 \pm 3.3 \mathrm{~kg}$ $(p=0.39)$ in the dapagliflozin and DPP4i groups, respectively (Table 6). The decrease in body weight was significantly greater in the dapagliflozin group than in the DPP4i group $(p<0.001)$. Similarly, BMI decreased significantly in the dapagliflozin group, but not in the DPP4i group from baseline to week 24: change from baseline was $-1.0 \pm 0.9 \mathrm{~kg} / \mathrm{m}^{2}(p<0.001)$ and $-0.1 \pm 1.2 \mathrm{~kg} / \mathrm{m}^{2} \quad(p=0.42) \quad$ in the 
Table 4 EQ-5D-5L

\begin{tabular}{|c|c|c|c|c|c|c|}
\hline & \multirow{2}{*}{ Week } & \multicolumn{2}{|c|}{ Dapagliflozin group } & \multicolumn{2}{|c|}{ DPP4i group } & \multirow{2}{*}{$\begin{array}{l}t \text { test } \\
p \text { value }\end{array}$} \\
\hline & & $n$ & Mean \pm SD & $n$ & Mean \pm SD & \\
\hline \multicolumn{7}{|l|}{ EQ-5D-5L score } \\
\hline \multirow[t]{2}{*}{ Score } & 0 & 123 & $0.927 \pm 0.110$ & 122 & $0.909 \pm 0.114$ & 0.23 \\
\hline & 24 & 109 & $0.938 \pm 0.096$ & 119 & $0.937 \pm 0.088$ & 0.95 \\
\hline Change from baseline & 24 & 109 & $\begin{array}{l}0.005 \pm 0.096 \\
(p=0.57)\end{array}$ & 117 & $\begin{array}{l}0.023 \pm 0.088 \\
(p=0.005)\end{array}$ & 0.14 \\
\hline \multicolumn{7}{|l|}{ EQ-5D-5L VAS } \\
\hline \multirow[t]{2}{*}{ Score } & 0 & 120 & $71.9 \pm 15.4$ & 121 & $70.6 \pm 17.1$ & 0.55 \\
\hline & 24 & 109 & $77.7 \pm 14.4$ & 120 & $76.5 \pm 12.5$ & 0.50 \\
\hline Change from baseline & 24 & 106 & $\begin{array}{l}4.9 \pm 16.1 \\
(p=0.002)\end{array}$ & 117 & $\begin{array}{l}5.4 \pm 14.1 \\
(p<0.001)\end{array}$ & 0.81 \\
\hline
\end{tabular}

Data are presented as mean \pm standard deviation $(\mathrm{SD})$; $p$ values for intragroup comparisons were obtained by using onesample $t$ test. Intergroup comparisons were performed by using two-sample $t$ test

$D P P 4 i$ dipeptidyl peptidase inhibitor, EQ-5D-5L EuroQol five-dimensional five-level version

dapagliflozin and DPP4i groups, respectively. The decrease in BMI was significantly greater in the dapagliflozin group $(p<0.001)$. Abdominal circumference decreased significantly in both groups from baseline to week 24: changes from baseline were $-2.7 \pm 4.3 \mathrm{~cm}(p<0.001)$ and $-1.2 \pm 4.8 \mathrm{~cm}(p=0.008)$ in the dapagliflozin and DPP4i groups, respectively. The decrease in abdominal circumference was significantly greater in the dapagliflozin group ( $p=0.019$ ).

HbA1c decreased significantly in both groups from baseline to week 24: changes from baseline were $\quad-0.8 \pm 1.1 \% \quad(p<0.001) \quad$ and $-0.9 \pm 1.4 \%(p<0.001)$ in the dapagliflozin and DPP4i groups, respectively. No significant intergroup difference was observed in the change in $\operatorname{HbA1c}(p=0.39)$.

\section{Correlation Analysis}

The proportion of subjects indicating improvement in the "overall quality of life" domain of SHIELD-WQ-9 at week 24 was significantly correlated with a decrease in body weight from baseline to week 24 (Spearman's rank correlation coefficient $\rho=-0.29,95 \%$ confidence interval $(\mathrm{CI})-0.40$ to $-0.16, p<0.001)$
(Table 7). The proportion of subjects indicating improvement in the "overall quality of life" domain of SHIELD-WQ-9 at week 24 was also significantly correlated with a decrease in HbA1c level from baseline to week 24 $(\rho=-0.20, \quad 95 \% \quad$ CI -0.33 to -0.07 , $p=0.003)$. Similarly, the proportion of subjects indicating improvement in the "physical health" domain was significantly correlated with a decrease in body weight from baseline to week $24(\rho=-0.39,95 \%$ CI -0.50 to -0.27 , $p<0.001)$ and with a decrease in HbA1c from baseline to week $24(\rho=-0.20,95 \%$ CI -0.32 to $-0.06, p=0.004)$. The change in DTR-QOL total score from baseline to week 24 was significantly correlated with a decrease in body weight from baseline to week $24(\rho=-0.17$, $95 \% \mathrm{CI}-0.29$ to $-0.04, p=0.012$ ) and with a decrease in HbA1c level from baseline to week $24(\rho=-0.21,95 \%$ CI -0.34 to -0.08 , $p=0.001)$. In contrast, the EQ-5D-5L score did not show any significant correlation with the decrease in body weight $(p=0.10)$ or HbA1c level from baseline to week $24(p=0.64)$. 
Table 5 Medication preference, medication adherence, and diet therapy adherence

\begin{tabular}{|c|c|c|c|c|c|c|c|}
\hline & \multirow[t]{2}{*}{ Week } & & \multicolumn{2}{|c|}{ Dapagliflozin group } & \multicolumn{2}{|c|}{ DPP4i group } & \multirow[t]{2}{*}{$p$ value } \\
\hline & & & $\bar{n}$ & $n(\%)$ & $n$ & $n(\%)$ & \\
\hline \multirow[t]{6}{*}{ Medication preference } & \multirow[t]{3}{*}{0} & Prefer SGLT2i & 119 & $22(18.5)$ & 120 & $15(12.5)$ & 0.007 \\
\hline & & Prefer DPP4i & & $6(5.0)$ & & $21(17.5)$ & \\
\hline & & Neither & & $91(76.5)$ & & $84(70.0)$ & \\
\hline & \multirow[t]{3}{*}{24} & Prefer SGLT2i & 106 & $38(35.8)$ & 117 & $10(8.5)$ & $<0.001$ \\
\hline & & Prefer DPP4i & & $6(5.7)$ & & $33(28.2)$ & \\
\hline & & Neither & & $62(58.5)$ & & $74(63.2)$ & \\
\hline Medication adherence & 24 & & 122 & $91.9 \pm 14.8$ & 123 & $94.1 \pm 9.6$ & 0.16 \\
\hline \multirow[t]{2}{*}{ Diet therapy adherence } & 0 & & 95 & $3.0[3.0,4.0]$ & 96 & $3.0[3.0,4.0]$ & 0.74 \\
\hline & 24 & & 95 & $3.0[3.0,4.0]$ & 106 & $3.0[3.0,4.0]$ & 0.56 \\
\hline
\end{tabular}

Data are presented as frequency (percentage), mean \pm standard deviation, and median [first quartile, third quartile] for medication preference, medication adherence, and diet therapy adherence, respectively. $p$ values for intergroup comparisons were obtained by using chi-square test, two-sample $t$ test, and Wilcoxon test for medication preference, medication adherence, and diet therapy adherence, respectively

$D P P 4 i$ dipeptidyl peptidase inhibitor, $S G L T 2 i$ sodium-glucose cotransporter 2 inhibitor

\section{Safety Outcomes}

During the study, 16 of 124 subjects in the dapagliflozin group (12.9\%) and 13 of 124 subjects in the DPP4i group (10.5\%) reported adverse events (Table 8). Death was not reported during the study, and serious adverse events were reported in $2(1.6 \%)$ and $1(0.8 \%)$ subjects in the dapagliflozin and DPP4i groups, respectively. One subject in the dapagliflozin group reported hypoglycemia-like symptoms, but the symptom was mild, and recovered without any treatment intervention. No severe hypoglycemia was reported in either group throughout the study. No significant difference was observed in the frequency of adverse events or serious adverse events between the groups. Known side effects of the study agents, such as dehydration, urinary tract infection, genital infection, and cystitis in the dapagliflozin group, and bullous pemphigoid in the DPP4i group, were not reported throughout the study.

\section{DISCUSSION}

The J-BOND study was conducted to compare the effects of an SGLT2i, dapagliflozin, and DPP4is on patients' QOL in Japanese patients with T2DM who had newly started therapy with OHAs. Since all subjects did not use insulin or GLP-1 receptor agonist at baseline, all subjects in this study were drug-naïve patients with T2DM. This was the first prospective randomized controlled trial to estimate the effects of SGLT2is and DPP4is on patients' QOL. Dapagliflozin showed comparable or even more favorable benefits on patients' QOL in this study, was well tolerated, and significantly reduced the body weight in the subjects. The reduction in body weight significantly correlated with the improvement of patients' QOL.

Although both DPP4i [18, 19] and SGLT2i [20-23] improve treatment satisfaction and QOL, no study has directly compared the effects of DPP4is and SGLT2is on patients' QOL. This was the first report to directly compare the effects of SGLT2i and DPP4is on patients' QOL, as measured by SHIELD-WQ-9. In this study, the proportion of subjects indicating improvement 
Table 6 Clinical outcomes

\begin{tabular}{|c|c|c|c|c|c|c|}
\hline \multirow[t]{2}{*}{ Variable } & \multirow[t]{2}{*}{ Week } & \multicolumn{2}{|c|}{ Dapagliflozin group } & \multicolumn{2}{|c|}{ DPP4i group } & \multirow{2}{*}{$\begin{array}{l}t \text { test } \\
p \text { value }\end{array}$} \\
\hline & & $n$ & Mean $\pm S D$ & $n$ & Mean \pm SD & \\
\hline \multicolumn{7}{|l|}{ Body weight (kg) } \\
\hline \multirow[t]{2}{*}{ Measurement } & 0 & 123 & $76.6 \pm 16.1$ & 124 & $76.4 \pm 15.3$ & 0.96 \\
\hline & 24 & 111 & $73.8 \pm 16.7$ & 120 & $75.6 \pm 13.4$ & 0.37 \\
\hline Change from baseline & 24 & 111 & $\begin{array}{l}-2.6 \pm 2.5 \\
(p<0.001)\end{array}$ & 120 & $\begin{array}{l}-0.3 \pm 3.3 \\
(p=0.39)\end{array}$ & $<0.001$ \\
\hline \multicolumn{7}{|l|}{$\operatorname{BMI}\left(\mathrm{kg} / \mathrm{m}^{2}\right)$} \\
\hline \multirow[t]{2}{*}{ Measurement } & 0 & 123 & $28.4 \pm 5.0$ & 124 & $28.3 \pm 4.5$ & 0.84 \\
\hline & 24 & 111 & $27.4 \pm 5.1$ & 120 & $27.9 \pm 4.0$ & 0.41 \\
\hline Change from baseline & 24 & 111 & $\begin{array}{l}-1.0 \pm 0.9 \\
(p<0.001)\end{array}$ & 120 & $\begin{array}{l}-0.1 \pm 1.2 \\
(p=0.42)\end{array}$ & $<0.001$ \\
\hline \multicolumn{7}{|c|}{ Abdominal circumference $(\mathrm{cm})$} \\
\hline \multirow[t]{2}{*}{ Measurement } & 0 & 113 & $97.2 \pm 12.3$ & 119 & $97.1 \pm 11.5$ & 0.95 \\
\hline & 24 & 104 & $95.0 \pm 12.8$ & 113 & $95.9 \pm 9.5$ & 0.56 \\
\hline Change from baseline & 24 & 99 & $\begin{array}{l}-2.7 \pm 4.3 \\
(p<0.001)\end{array}$ & 109 & $\begin{array}{l}-1.2 \pm 4.8 \\
(p=0.008)\end{array}$ & 0.019 \\
\hline \multicolumn{7}{|l|}{ HbAlc (\%) } \\
\hline \multirow[t]{2}{*}{ Measurement } & 0 & 119 & $7.8 \pm 1.4$ & 120 & $8.0 \pm 1.6$ & 0.48 \\
\hline & 24 & 115 & $6.9 \pm 0.7$ & 121 & $7.0 \pm 1.1$ & 0.22 \\
\hline Change from baseline & 24 & 110 & $\begin{array}{l}-0.8 \pm 1.1 \\
(p<0.001)\end{array}$ & 117 & $\begin{array}{l}-0.9 \pm 1.4 \\
(p<0.001)\end{array}$ & 0.39 \\
\hline
\end{tabular}

Data are presented as mean \pm standard deviation $(\mathrm{SD}) . p$ values for intragroup comparisons were obtained by using onesample $t$ test. Intergroup comparisons were performed by using two-sample $t$ test

DPP4i dipeptidyl peptidase inhibitor

in the "overall quality of life" domain of SHIELD-WQ-9 at week 24 was higher in the dapagliflozin group than in the DPP4i group. Body weight significantly decreased in the dapagliflozin group compared with the DPP4i group, and the proportion of subjects indicating improvement in the "overall quality of life" domain of SHIELD-WQ-9 at week 24 significantly correlated with the decrease in body weight from baseline to week 24. Similarly, the proportion of subjects indicating improvement in the "physical health" domain of SHIELD-
WQ-9 at week 24 was significantly higher in the dapagliflozin group, and it significantly correlated with the decrease in body weight from baseline to week 24. As SHIELD-WQ-9 measures the perceived benefit of weight control [24], these results suggest the benefit of dapagliflozin on patients' QOL via the body weight reduction. Previous studies have also demonstrated a greater proportion of subjects treated with dapagliflozin reporting improvement in overall QOL [20] or physical health [28] in SHIELDWQ-9 than those treated with the placebo. 
Table 7 Correlation analysis

\begin{tabular}{|c|c|c|c|c|c|c|}
\hline \multirow[t]{3}{*}{ Variable 1} & \multicolumn{6}{|c|}{ Variable 2} \\
\hline & \multicolumn{3}{|c|}{$\begin{array}{l}\text { Change in body weight from } \\
\text { baseline to week } 24\end{array}$} & \multicolumn{3}{|c|}{$\begin{array}{l}\text { Change in HbA1c from } \\
\text { baseline to week } 24\end{array}$} \\
\hline & $\bar{n}$ & $\rho(95 \% \mathrm{CI})$ & $p$ value & $n$ & $\rho(95 \% \mathrm{CI})$ & $p$ value \\
\hline $\begin{array}{l}\text { Improvement in "overall quality of life" domain of } \\
\text { SHIELD-WQ-9 at week } 24\end{array}$ & 225 & $\begin{array}{l}-0.29(-0.40 \\
-0.16)\end{array}$ & $<0.001$ & 218 & $\begin{array}{l}-0.20(-0.33 \\
-0.07)\end{array}$ & 0.003 \\
\hline $\begin{array}{l}\text { Improvement in "physical health" domain of } \\
\text { SHIELD-WQ-9 at week } 24\end{array}$ & 225 & $\begin{array}{l}-0.39(-0.50 \\
-0.27)\end{array}$ & $<0.001$ & 218 & $\begin{array}{l}-0.20(-0.32 \\
-0.06)\end{array}$ & 0.004 \\
\hline $\begin{array}{l}\text { Change in DTR-QOL total score from baseline to } \\
\text { week } 24\end{array}$ & 222 & $\begin{array}{l}-0.17(-0.29 \\
-0.04)\end{array}$ & 0.012 & 216 & $\begin{array}{l}-0.21(-0.34 \\
-0.08)\end{array}$ & 0.001 \\
\hline $\begin{array}{l}\text { Change in EQ-5D-5L score from baseline to } \\
\text { week } 24\end{array}$ & 224 & $\begin{array}{l}-0.11(-0.24 \\
0.02)\end{array}$ & 0.10 & 218 & $\begin{aligned} &-0.03(-0.16 \\
&0.10)\end{aligned}$ & 0.64 \\
\hline
\end{tabular}

Data are represented as Spearman's rank correlation coefficient $(\rho)$ and $95 \%$ confidence interval (CI) DTR-QOL Diabetes Therapy-Related Quality of Life, EQ-5D-5L EuroQol five-dimensional five-level version, SHIELD$W Q-9$ Study to Help Improve Early evaluation and management of risk factors Leading to Diabetes Weight Questionnaire9

There are no reports on the effects of DPP4is on weight-change-related QOL using SHIELD-WQ9. As DPP4is had a neutral effect on body weight in this study, we presumed that DPP4is did not have much impact on SHIELD-WQ-9 owing to any change in body weight.

The total scores and domain 1 scores of DTRQOL improved in the dapagliflozin group compared with those in the DPP4i group. The change in DTR-QOL total scores from baseline to week 24 significantly correlated with the decrease in body weight from baseline to week 24 in all subjects. These results also suggest that the body weight reduction by dapagliflozin contributed to the improvement in the patients' QOL. DTR-QOL is a diabetes-specific QOL questionnaire [25]. Previous studies have demonstrated that both SGLT2is $[29,30]$ and DPP4is [31, 32] improve scores in DTR-QOL. The results of this study showing a significant improvement in DTR-QOL scores in both groups were consistent with the findings of previous reports. However, this is the first report to directly compare the effects of SGLT2i and DPP4is on DTR-QOL and show the more favorable impact of dapagliflozin on diabetes therapy-related QOL compared with the DPP4is.
The proportion of subjects indicating improvement in the "overall quality of life" and "physical health" domains of SHIELD-WQ-9 at week 24 and change in total scores of DTR-QOL from baseline to week 24 significantly correlated with the decrease in HbA1c from baseline to week 24. Both dapagliflozin and DPP4is significantly and comparably improved the HbA1c in the study subjects. These results suggest that the beneficial effects of dapagliflozin and DPP4is on the patients' QOL by improving glycemic control were comparable.

Although the DPP4i group showed a significant increase in the EQ-5D-5L scores, no significant intergroup difference was found in the change in EQ-5D-5L scores and VAS. Although previous studies have shown that neither dapagliflozin [21] nor DPP4is [33, 34] improved EQ-5D scores compared with the placebo, only one study using sitagliptin showed a significant increase in EQ-5D scores [35]. Although the results for EQ-5D-5L scores in this study were consistent with those of previous reports, this was the first report to directly compare the effects of SGLT2i and DPP4is on EQ-5D-5L scores and demonstrate that dapagliflozin and 
Table 8 Adverse events

\begin{tabular}{|c|c|c|c|}
\hline \multirow[t]{2}{*}{ Adverse event } & \multicolumn{2}{|l|}{$n(\%)$} & \multirow{2}{*}{$\begin{array}{l}\text { Fisher's exact test } \\
p \text { value }\end{array}$} \\
\hline & $\overline{\text { Dapagliflozin group }}$ & $\overline{\text { DPP4i group }}$ & \\
\hline Number of subjects in the safety analysis set & 124 & 124 & - \\
\hline Death & $0(0.0)$ & $0(0.0)$ & - \\
\hline Any adverse event & $16(12.9)$ & $13(10.5)$ & 0.69 \\
\hline Serious adverse event & $2(1.6)$ & $1(0.8)$ & 1.00 \\
\hline Nausea & $1(0.8)$ & $0(0.0)$ & 1.00 \\
\hline Pharyngitis & $2(1.6)$ & $1(0.8)$ & 1.00 \\
\hline Hypoesthesia & $0(0.0)$ & $1(0.8)$ & 1.00 \\
\hline Elevated hepatic enzymes & $0(0.0)$ & $1(0.8)$ & 1.00 \\
\hline Periarthritis & $0(0.0)$ & $1(0.8)$ & 1.00 \\
\hline Bronchitis & $0(0.0)$ & $1(0.8)$ & 1.00 \\
\hline Balanoposthitis & $1(0.8)$ & $0(0.0)$ & 1.00 \\
\hline Myringitis & $1(0.8)$ & $0(0.0)$ & 1.00 \\
\hline Hyperglycemia & $0(0.0)$ & $1(0.8)$ & 1.00 \\
\hline Bone fracture & $2(1.6)$ & $2(1.6)$ & 1.00 \\
\hline Lipoma & $0(0.0)$ & $1(0.8)$ & 1.00 \\
\hline Tooth injury & $0(0.0)$ & $1(0.8)$ & 1.00 \\
\hline Upper abdominal pain & $0(0.0)$ & $1(0.8)$ & 1.00 \\
\hline Myocardial infarction & $1(0.8)$ & $0(0.0)$ & 1.00 \\
\hline Herpes zoster & $2(1.6)$ & $0(0.0)$ & 0.50 \\
\hline Enteritis & $1(0.8)$ & $0(0.0)$ & 1.00 \\
\hline Intervertebral disc extrusion & $1(0.8)$ & $0(0.0)$ & 1.00 \\
\hline Hypoglycemia-like syndrome & $1(0.8)$ & $0(0.0)$ & 1.00 \\
\hline Palpitation & $1(0.8)$ & $0(0.0)$ & 1.00 \\
\hline Ureterolithiasis & $0(0.0)$ & $1(0.8)$ & 1.00 \\
\hline Skin rash & $1(0.8)$ & $1(0.8)$ & 1.00 \\
\hline Frequent urination & $2(1.6)$ & $0(0.0)$ & 0.50 \\
\hline Tachycardia & $1(0.8)$ & $0(0.0)$ & 1.00 \\
\hline Dizziness & $1(0.8)$ & $0(0.0)$ & 1.00 \\
\hline Sinusitis & $1(0.8)$ & $0(0.0)$ & 1.00 \\
\hline Constipation & $2(1.6)$ & $2(1.6)$ & 1.00 \\
\hline Peripheral neuropathy & $0(0.0)$ & $1(0.8)$ & 1.00 \\
\hline
\end{tabular}

$D P P 4 i$ dipeptidyl peptidase inhibitor 
DPP4is had comparable impact on patients' QOL, as measured by EQ-5D-5L scores.

The administration of SGLT2i reduces body weight [20, 36-38], whereas DPP4i has a neutral effect on body weight [39-41]. The results of this study were consistent with those of previous reports: body weight and BMI significantly decreased in the dapagliflozin group, but not in the DPP4i group. Since no rebound of body weight was observed in this study (dapagliflozin group: $\quad 76.6 \pm 16.1, \quad 74.1 \pm 16.1, \quad$ and $73.8 \pm 16.7 \mathrm{~kg}$ at baseline, week 12 , and 24 . DPP4i group: $76.4 \pm 15.3,75.6 \pm 13.4$, and $75.6 \pm 13.4 \mathrm{~kg}$ at baseline, week 12 , and 24 ), it may not affect the PROs. Although a significant decrease in abdominal circumference was observed in both groups, it was significantly greater in the dapagliflozin group. As significant correlations were found between the decrease in body weight and SHIELD-WQ-9 or DTR-QOL scores, the favorable effects of dapagliflozin on body weight may have contributed to the improvement in patients' QOL.

No remarkable safety concern was observed in either group throughout the study. Only one hypoglycemia-like symptom was reported in the dapagliflozin group, but the symptom was mild, and recovered without any treatment intervention. Known side effects of the study agents, such as dehydration, urinary tract infection, genital infection, and cystitis in the dapagliflozin group, and bullous pemphigoid in the DPP4i group, were not reported throughout the study. This may be the reason why the domain 2 score of DTR-QOL for "anxiety and dissatisfaction with treatment" improved in both groups, and no significant intergroup difference was observed in the change in domain 2 scores of DTR-QOL.

Probably because both dapagliflozin and the DPP4is improved patients' QOL, the subjects' responses in both groups indicated that they preferred the assigned study agent. Medication adherence for the study agent was comparable in both groups. A previous study comparing treatment satisfaction and medication adherence among DPP4is, $\alpha$-glucosidase inhibitors, biguanides, and sulfonylureas showed that the DPP4i group had the highest treatment satisfaction and adherence, comparable HbA1c- lowering effects, and fewer adverse events [19]. DPP4is were the most preferable option in terms of treatment satisfaction. Because the previous study was conducted before the launch of SGLT2is in Japan, it did not compare medication adherence for SGLT2is. In this study, medication adherence in the dapagliflozin group was relatively high $(91.9 \pm 14.8 \%)$ and comparable to that in the DPP4i group in this study $(94.1 \pm 9.6 \%)$ and to that in the DPP4i group in the previous study (92.9\%) [19].

Altogether, dapagliflozin showed a comparable or even more favorable benefit on patients' QOL compared with that of DPP4is, with comparable medication adherence, HbA1c reduction, and frequency of adverse events including hypoglycemia. These results suggest that dapagliflozin can be used as a comparable or superior antidiabetic agent compared with DPP4is.

This study has several limitations. First, this was an open-label trial, and the primary endpoint and the major secondary endpoints were PROs. Therefore, the study design as the openlabel trial could be a cause of bias to the PROs. Second, all the subjects in this study were Japanese. Although SGLT2is and DPP4is are available worldwide, the results of this study cannot be generalized to patients with T2DM in other countries. In addition, DPP4is are more effective in East Asian patient populations compared with non-Asian patients [6]. As the change in HbA1c was significantly correlated with the improvement in SHIELD-WQ-9 and DTR-QOL scores in this study, the beneficial impact of DPP4is on patients' QOL may differ between Asian and non-Asian patients. Further study is required to compare the benefit of SGLT2is and DPP4is on patients' QOL in other Asian and non-Asian countries. Third, this study enrolled drug-naïve patients with T2DM who did not know the effects of other OHAs, and whose responses regarding medication preference were mainly based on the explanation of mode of action, effects, advantages, and possible side effects by the investigators. The effects of SGLT2is and DPP4is on patients' QOL and medication preference should be further investigated upon the switch or add-on of other OHAs. 


\section{CONCLUSION}

Dapagliflozin showed comparable or even more favorable benefit on patients' QOL compared with the DPP4is. Dapagliflozin was well tolerated, and it significantly reduced body weight. The reduction in body weight significantly correlated with the improvement in patients' QOL. In concordance with the recommendation of a "patient-centered approach" by the ADA/EASD $[1,2,17]$ for developing a treatment strategy, our results demonstrate that dapagliflozin can be used as a first-line drug for T2DM in Japan with a beneficial impact on patients' QOL.

\section{ACKNOWLEDGEMENTS}

The authors thank all of the clinical staff for their assistance in the execution of the study. We thank all the participants of this study.

Funding. This study, the technical assistance in the launch and execution of the study and the journal's Rapid Service Fee were funded by AstraZeneca K.K.

Medical Writing and Other Assistance. The authors thank Arata Yoneda of Soiken Inc. for their technical assistance in the launch and execution of the study and support on the medical writing of the manuscript. This assistance was funded by AstraZeneca K.K.

Authorship. All named authors meet the International Committee of Medical Journal Editors (ICMJE) criteria for authorship for this article, take responsibility for the integrity of the work as a whole, and have given their approval for this version to be published.

Authorship Contributions. HI contributed to the conception, design of the study, subject enrollment, data acquisition, drafting and revision of the manuscript. NK, TN, and DS contributed to the design of the study, subject enrollment, and data acquisition. HN, TH, YH, $\mathrm{SO}$, and TN contributed to subject enrollment and data acquisition. All named authors meet the International Committee of Medical Journal Editors (ICMJE) criteria for authorship for this article, take responsibility for the integrity of the work as a whole, and have given their approval for this version to be published.

Disclosures. Hitoshi Ishii has received honoraria from Ono Pharmaceutical Co., Mitsubishi Tanabe Pharma, MSD, Novo Nordisk Pharma Ltd., Daiichi Sankyo, Takeda Pharmaceutical Co., Eli Lilly Japan, Sanofi, and Sumitomo Dainippon Pharma Co., Ltd. Nozomu Kamei has received lecture fee from Sumitomo Dainippon Pharma Co., Ltd., and a grant from Astellas Pharma Inc. DS has received lecture fees from Ono Pharmaceutical Co., MSD K.K., Sumitomo Dainippon Pharma Co., Ltd., Eli Lilly and Company, Boehringer Ingelheim Pharmaceuticals, Inc., Takeda Pharmaceutical Co., Ltd., and Taisho Toyama Pharmaceutical Co., Ltd. Hiroki Nakajima, Tetsuji Niiya, Toru Hiyoshi, Yuko Hiramori, Shigeyuki Ohtsu, Takashi Noto and Dai Shimono have nothing to disclose.

Compliance with Ethic Guidelines. This study and its protocols were first approved by the institutional review board of each participating institution (Supplementary Material), according to the Ethical Guidelines for Medical and Health Research Involving Human Subjects issued by the Ministry of Health, Labour and Welfare in Japan. Following the enforcement of the Clinical Trials Act in April 2018, this study and its protocols were again inspected and approved by the Nara Medical University Certified Review Board, which had obtained certification from the Minister of Health, Labour and Welfare in Japan. This study was first registered in the University Hospital Medical Information Network Clinical Trial Registry (UMIN-CTR) (registration number UMIN000030514) and then in the Japan Registry of Clinical Trials (jRCT) (registration number jRCTs051180165) after the approval from the certified review board according to the requirement in the Clinical Trials Act. The study was conducted in accordance with the Declaration of Helsinki, Ethical Guidelines for Medical and Health Research Involving Human 
Subject, the Clinical Trials Act, and other current legal regulations in Japan. Written informed consent was obtained prior to treatment from all enrolled patients who met the eligibility criteria.

Data Availability. The datasets generated during and/or analyzed during the current study are not publicly available due to the lack of a statement in the study protocol enabling data sharing with a third party after the end of the study and in the informed consent documents as well as lack of approval for data sharing by the institutional review board of each participating institution or the Nara Medical University Certified Review Board.

Open Access. This article is licensed under a Creative Commons Attribution-NonCommercial 4.0 International License, which permits any non-commercial use, sharing, adaptation, distribution and reproduction in any medium or format, as long as you give appropriate credit to the original author(s) and the source, provide a link to the Creative Commons licence, and indicate if changes were made. The images or other third party material in this article are included in the article's Creative Commons licence, unless indicated otherwise in a credit line to the material. If material is not included in the article's Creative Commons licence and your intended use is not permitted by statutory regulation or exceeds the permitted use, you will need to obtain permission directly from the copyright holder. To view a copy of this licence, visit http://creativecommons.org/licenses/by$\mathrm{nc} / 4.0 /$.

\section{REFERENCES}

1. Davies MJ, D'Alessio DA, Fradkin J, et al. Management of hyperglycemia in type 2 diabetes, 2018. A consensus report by the American Diabetes Association (ADA) and the European Association for the study of diabetes (EASD). Diabetes Care. 2018;41: 2669-701.

2. Buse JB, Wexler DJ, Tsapas A, et al. 2019 Update to: management of hyperglycemia in type 2 diabetes, 2018. A consensus report by the American Diabetes
Association (ADA) and the European Association for the study of diabetes (EASD). Diabetes Care. 2020;43:487-93.

3. Haneda $M$, Noda $M$, Origasa $H$, et al. Japanese clinical practice guideline for diabetes 2016. Diabetol Int. 2018;9:1-45.

4. The Japan Diabetes Society (2020) Japanese Clinical Practice Guideline for Diabetes 2020-2021. Bunkodo.

5. Karagiannis T, Boura P, Tsapas A. Safety of dipeptidyl peptidase 4 inhibitors: a perspective review. Ther Adv Drug Saf. 2014;5:138-46.

6. Kim YG, Hahn S, Oh TJ, Kwak SH, Park KS, Cho YM. Differences in the glucose-lowering efficacy of dipeptidyl peptidase-4 inhibitors between Asians and non-Asians: a systematic review and metaanalysis. Diabetologia. 2013;56:696-708.

7. Seino Y, Kuwata H, Yabe D. Incretin-based drugs for type 2 diabetes: focus on East Asian perspectives. J Diabetes Investig. 2016;7:102-9.

8. Bolinder J, Ljunggren O, Kullberg J, et al. Effects of dapagliflozin on body weight, total fat mass, and regional adipose tissue distribution in patients with type 2 diabetes mellitus with inadequate glycemic control on metformin. J Clin Endocrinol Metab. 2012;97:1020-31.

9. Dandona P, Chaudhuri A. Sodium-glucose cotransporter 2 inhibitors for type 2 diabetes mellitus: an overview for the primary care physician. Int J Clin Pract. 2017;71:e12937.

10. Zinman B, Wanner C, Lachin JM, et al. Empagliflozin, cardiovascular outcomes, and mortality in type 2 diabetes. N Engl J Med. 2015;373:2117-288.

11. Wanner C, Inzucchi SE, Zinman B. Empagliflozin and progression of kidney disease in type 2 diabetes. N Engl J Med. 2016;375:1801-2.

12. Neal B, Perkovic V, Mahaffey KW, et al. Canagliflozin and cardiovascular and renal events in type 2 diabetes. N Engl J Med. 2017;377:644-57.

13. Mahaffey KW, Neal B, Perkovic V, et al. Canagliflozin for primary and secondary prevention of cardiovascular events: results from the CANVAS program (canagliflozin cardiovascular assessment study). Circulation. 2017;137:323-34.

14. Li J, Gong Y, Li C, Lu Y, Liu Y, Shao Y. Long-term efficacy and safety of sodium-glucose cotransporter2 inhibitors as add-on to metformin treatment in the management of type 2 diabetes mellitus: a meta-analysis. Medicine (Baltimore). 2017;96: e7201. 
15. Kohler S, Zeller C, Iliev H, Kaspers S. Safety and tolerability of empagliflozin in patients with type 2 diabetes: pooled analysis of phase I-III clinical trials. Adv Ther. 2017;34:1707-26.

16. Schernthaner G, Gross JL, Rosenstock J, et al. Canagliflozin compared with sitagliptin for patients with type 2 diabetes who do not have adequate glycemic control with metformin plus sulfonylurea: a 52-week randomized trial. Diabetes Care. 2013;36: 2508-15.

17. Inzucchi SE, Bergenstal RM, Buse JB, et al. Management of hyperglycaemia in type 2 diabetes, 2015: a patient-centred approach. Update to a position statement of the American Diabetes Association and the European Association for the Study of Diabetes. Diabetes Care. 2015;38:140-9.

18. Davies M, Speight J. Patient-reported outcomes in trials of incretin-based therapies in patients with type 2 diabetes mellitus. Diabetes Obes Metab. 2012;14:882-92.

19. Ishii H, Hayashino Y, Akai Y, Yabuta M, Tsujii S. Dipeptidyl peptidase-4 inhibitors as preferable oral hypoglycemic agents in terms of treatment satisfaction: results from a multicenter, 12-week, open label, randomized controlled study in Japan (PREFERENCE 4 study). J Diabetes Investig. 2018;9: 137-45.

20. Grandy S, Hashemi M, Langkilde AM, Parikh S, Sjostrom CD. Changes in weight loss-related quality of life among type 2 diabetes mellitus patients treated with dapagliflozin. Diabetes Obes Metab. 2014;16:645-50.

21. Grandy S, Langkilde AM, Sugg JE, Parikh S, Sjostrom CD. Health-related quality of life (EQ-5D) among type 2 diabetes mellitus patients treated with dapagliflozin over 2 years. Int J Clin Pract. 2014;68: 486-94.

22. Bolge SC, Flores NM, Huang S, Cai J. Health care provider experience with canagliflozin in real-world clinical practice: favorability, treatment patterns, and patient outcomes. Int J Gen Med. 2017;10: 177-87.

23. Nakajima H, Okada S, Mohri T, et al. Dapagliflozin improves treatment satisfaction in overweight patients with type 2 diabetes mellitus: a patient reported outcome study (PRO study). Diabetol Metab Syndr. 2018;10:11.

24. Grandy S, Fox KM, Bazata DD. Association of selfreported weight change and quality of life, and exercise and weight management behaviors among adults with type 2 diabetes mellitus: the SHIELD study. Cardiol Res Pract. 2012;2012:892564.
25. Ishii H. Development and psychometric validation of the Diabetes Therapy-Related QOL (DTR-QOL) questionnaire. J Med Econ. 2012;15:556-63.

26. Herdman M, Gudex C, Lloyd A, et al. Development and preliminary testing of the new five-level version of EQ-5D (EQ-5D-5L). Qual Life Res. 2011;20: 1727-36.

27. Shiroiwa T, Ikeda $S$, Noto $S$, et al. Comparison of value set based on DCE and/or TTO data: scoring for EQ-5D-5L health states in Japan. Value Health. 2016;19:648-54.

28. Grandy S, Sternhufvud C, Ryden A, Sugg J, Rohwedder K. Patient-reported outcomes among patients with type 2 diabetes mellitus treated with dapagliflozin in a triple-therapy regimen for 52 weeks. Diabetes Obes Metab. 2016;18:306-9.

29. Yamazaki M, Higo N, Kaneko T, et al. SGLT2 inhibitors as the trigger for diabetes care: reconfirmed importance of behavior modification after drug administration. J Jpn Diabetes Soc. 2015;58:745-52.

30. Yoshikawa F, Kumashiro N, Shigiyama F, et al. Efficacy of intermittent empagliflozin supplementation on dietary self-management and glycaemic control in patients with poorly controlled type 2 diabetes: a 24-week randomized controlled trial. Diabetes Obes Metab. 2019;21:303-11.

31. Ishii H, Suzaki Y, Miyata Y, Matsui S. Randomized multicenter evaluation of quality of life and treatment satisfaction in type 2 diabetes patients receiving once-weekly trelagliptin versus a daily dipeptidyl peptidase-4 inhibitor. Diabetes Ther. 2019;10:1369-80.

32. Mita T, Katakami N, Shiraiwa T, et al. The influence of sitagliptin on treatment-related quality of life in patients with type 2 diabetes mellitus receiving insulin treatment: a prespecified sub-analysis. Diabetes Ther. 2017;8:693-704.

33. Briggs AH, Bhatt DL, Scirica BM, et al. Health-related quality-of-life implications of cardiovascular events in individuals with type 2 diabetes mellitus: a subanalysis from the Saxagliptin assessment of vascular outcomes recorded in patients with diabetes mellitus (SAVOR)-TIMI 53 trial. Diabetes Res Clin Pract. 2017;130:24-33.

34. Sakamoto Y, Oyama J, Ikeda H, et al. Effects of sitagliptin beyond glycemic control: focus on quality of life. Cardiovasc Diabetol. 2013;12:35.

35. Best JH, Rubin RR, Peyrot M, et al. Weight-related quality of life, health utility, psychological wellbeing, and satisfaction with exenatide once weekly compared with sitagliptin or pioglitazone after 26 weeks of treatment. Diabetes Care. 2011;34:314-9. 
36. Ribola FA, Cancado FB, Schoueri JH, De Toni VF, Medeiros VH, Feder D. Effects of SGLT2 inhibitors on weight loss in patients with type 2 diabetes mellitus. Eur Rev Med Pharmacol Sci. 2017;21: 199-21111.

37. Hirose S, Nakajima S, Iwahashi Y, Seo A, Takahashi T, Tamori Y. Impact of the 8-week administration of tofogliflozin for glycemic control and body composition in Japanese patients with type 2 diabetes mellitus. Intern Med. 2016;55:3239-45.

38. Tosaki T, Kamiya H, Himeno T, et al. Sodium-glucose co-transporter 2 inhibitors reduce the abdominal visceral fat area and may influence the renal function in patients with type 2 diabetes. Intern Med. 2017;56:597-604.
39. Umezawa S, Kubota A, Maeda H, et al. Two-year assessment of the efficacy and safety of sitagliptin in elderly patients with type 2 diabetes: post hoc analysis of the ASSET-K study. BMC Endocr Disord. 2015;15:34.

40. Hermansen K, Kipnes M, Luo E, Fanurik D, Khatami $H$, Stein P. Efficacy and safety of the dipeptidyl peptidase- 4 inhibitor, sitagliptin, in patients with type 2 diabetes mellitus inadequately controlled on glimepiride alone or on glimepiride and metformin. Diabetes Obes Metab. 2007;9:733-45.

41. Katsuno T, Ikeda H, Ida K, Miyagawa J, Namba M. Add-on therapy with the DPP-4 inhibitor sitagliptin improves glycemic control in insulin-treated Japanese patients with type 2 diabetes mellitus. Endocr J. 2013;60:733-42. 